\title{
Absorption, Distribution, Metabolism, and Excretion (ADME) of Therapeutic Proteins: Current Industry Practices and Future Perspectives
}

Jayaprakasam Bolleddula, Kevin Brady, Gerard Bruin, Anthony Lee, Jennifer A Martin, Markus Walles, Keyang Xu, Tong-Yuan Yang, Xiaochun Zhu, and Hongbin Yu

Quantitative Pharmacology, EMD Serono Research \& Development Institute, Inc., Billerica, Massachusetts, USA (J.B.); Quantitative Pharmacology \& DMPK, UCB Pharma S.A., Slough, UK (K.B.); Pharmacokinetic Science, Novartis Institutes for Biomedical Research, Basel, Switzerland (G.B., M.W.); Quantitative Pharmacology and Disposition, Seagen Inc., Bothell, WA, USA (A. L.); Drug Disposition, Eli Lilly and Company, Indianapolis, IN 46285, USA (J. A. M); BioAnalytical Sciences, Genentech, South San Francisco, CA, USA (K. X.); Preclinical Sciences and Translational Safety, Janssen R\&D, LLC., Spring House, PA, USA (T-Y. Y.); Drug Metabolism and Pharmacokinetics, Takeda Pharmaceutical Company Limited, Cambridge, MA, USA (X. Z.); Drug Metabolism and Pharmacokinetics, Boehringer Ingelheim Pharmaceuticals, Inc., Ridgefield, CT, USA (H. Y.) 
Running Title: ADME of therapeutic proteins

Address Correspondence to: Jayaprakasam Bolleddula, Quantitative Pharmacology, EMD Serono Research \& Development Institute, Inc., 45 A Middlesex Turnpike, Billerica, Massachusetts, USA. E-mail: jayaprakasam.bolleddual@emdserono.com; Kevin Brady, Cytoseek Ltd, Science Creates Old Market, Bristol, UK. E-mail: kevin@ cytoseek.uk.

Text pages: 20

Tables: 2

Figures: 6

References: 68

Abstract: 239

Introduction: 824

Discussion: 5131 


\section{Nonstandard Abbreviations}

TPs, therapeutic proteins; WG, working group; TALG, translational and ADME sciences leadership group; IQ, innovation and quality; NCE, new chemical entity; ICH, international consortium for harmonisation; BLA, biologics license application; PK/PD, pharmacokinetics/pharmacodynamics; FcRn, neonatal Fc receptor; SC, subcutaneous; IV, intravenous; FcRn KO, neonatal Fc receptor knock-out; SCID, severe combined immunodeficient; ADA, anti-drug antibody; NHP, non-human primates; TMDD, target meditated drug disposition; QWBA, quantitative whole body autoradiography; PET, positron emission tomography; SPECT, single-photon emission computed tomography; CT, computed tomography; MALDI-MS, matrix-assisted laser desorption ionization-mass spectrometry; CSF, cerebrospinal fluid; CNS, central nervous system; PBPK, physiologically based pharmacokinetic; TNF, tumor necrosis factor; IgG, immunoglobulin G; EGFR, epidermal growth factor receptor; PTM, post translational modification; ADC, antibody-drug conjugate; GLP-1, glucagon-like peptide 1; GIP, gastric inhibitory polypeptide; MW, molecular weight; FGF21, fibroblast growth factor 21; BP, biotransformation product; LBA, ligand binding assay. 


\begin{abstract}
Therapeutics proteins (TPs) comprise a variety of modalities including antibody-based drugs, coagulation factors, recombinant cytokines, enzymes, growth factors, and hormones. TPs usually cannot traverse cellular barriers and exert their pharmacological activity by interacting with targets on the exterior membrane of cells or with soluble ligands in the tissue interstitial fluid/blood. Due to large size, lack of cellular permeability, variation in metabolic fate, and distinct physicochemical characteristics, TPs are subject to different absorption, distribution, metabolism, and excretion (ADME) processes as compared to small molecules. Limited regulatory guidance makes it challenging to determine the most relevant ADME data required for regulatory submissions. The TP ADME working group (WG) was sponsored by the Translational and ADME Sciences Leadership Group (TALG) within the Innovation and Quality (IQ) consortium with objectives to: i) better understand the current practices of ADME data generated for TPs across IQ member companies, ii) learn about their regulatory strategy and interaction experiences, and iii) provide recommendations on best practices for conducting ADME studies. To understand current ADME practices and regulatory strategies, an industry-wide survey was conducted within IQ member companies. In addition, ADME data submitted to FDA was also collated by reviewing regulatory submission packages of TPs approved between 2011-2020. This article summarizes the key learnings from the survey and an overview of ADME data presented in BLAs along with future perspectives and recommendations for conducting ADME studies for internal decision making as well as regulatory submissions for TPs.
\end{abstract}




\section{SIGNIFICANCE STATEMENT}

This article provides comprehensive assessment of the current practices of absorption, distribution, metabolism, and excretion (ADME) data generated for therapeutic proteins across the Innovation and Quality (IQ) participating companies and the utility of the data in discovery, development, and regulatory submissions. The TP ADME working group (WG) working group also recommends the best practices for conducting ADME studies for internal decision making and regulatory submissions. 


\section{Introduction}

During drug discovery and development, candidate molecules are iteratively screened for drug-like properties, with a view to optimize key parameters associated with Absorption, Distribution, Metabolism and Excretion (ADME) (Doogue and Polasek, 2013). The goal of optimization is to develop compounds that can maintain required exposures to attain expected pharmacological activity, while minimizing potential toxicities. As compounds advance towards human clinical trials, key aspects of the ADME characteristics need to be scrutinized and presented for regulatory review. An understanding of physiological processes that influence drug exposure, safety and efficacy come under the spotlight for compounds at this stage.

One common feature explored in small molecule drug discovery is the ability of the compound to cross cellular membranes as the target for pharmacology is often present intracellularly. It also contributes for understanding off-target toxic effects, a phenomenon often encountered with small molecule drugs. Even for cases where the pharmacological target is located on the membrane surface of a cell, or soluble in the tissue interstitial fluid, the compound still needs to be absorbed from the gut (Absorption) or distributed to the target tissue of interest. One consequence of this permeability is the exposure of the drug to metabolizing enzymes present in cells, primarily in the liver and the gut (Metabolism). Compounds are also subject to transporter activity that can further perturb the equilibrium of drug concentration inside and outside of cells, or between the systemic circulation and extravascular space (Distribution and/or Elimination). Over the last 20 years, a significant amount of regulatory guidance related to ADME of new chemical entities (NCEs) has been issued (Supplementary Table 1).

The world of TPs is extremely diverse, from cytokine proteins and peptides with molecular weights (MWs) of 5-15 kDa up to antibodies $(\sim 150 \mathrm{kDa})$ or larger. Generally, TPs are 
proteins that are produced in cell culture (mammalian, plant, yeast or bacterial) systems using recombinant/genetic engineering technologies. An overview of different types of TPs is shown in Fig. 1.

TPs, by contrast to NCEs, are typically not readily permeable, and generally may not need to enter the cytoplasm of cells to exert their pharmacological effect. However, it is important to emphasize that proteins can indeed enter cells, through mechanisms such as pinocytosis or receptor-mediated endocytosis. They can exert their biochemical and pharmacological actions in endosomal or lysosomal compartments. TPs can also undergo salvage from metabolism within these compartments, exemplified by rescue of antibody therapeutics by the neonatal Fc Receptor, FcRn, leading to longer half-life. However, their ability to escape from these vesicles into the cytoplasm of the cell is significantly limited by their size and physicochemical complex nature. They do not therefore directly engage with the classical metabolic pathways of small molecules (i.e. cytochrome P450s). Instead, they are subject to intracellular degradation within lysosomes or by proteolytic cleavage in interstitial fluids/blood. As a result of this lack of permeability and the physicochemical nature of protein therapeutics, they are subject to distinctly different ADME processes, similar to how endogenous proteins are metabolized by the body. A number of reviews describing the differing physicochemical properties and ADME behaviours of TPs have been published (Brady and Webster, 2012; Hamuro and Kishnani, 2012; Tibbitts et al., 2016; Conner et al., 2020). In contrast to small molecules, considerably less regulatory guidance is available for TPs.

The diversity in molecular structure of TPs and their associated ADME behaviors has made it challenging to apply a standard set of studies for their discovery and development. The mantra for studying ADME of TPs is often stated as 'case-by-case', with limited guidance 
defining the required studies for ADME regulatory packages. For most TPs, the guidance for preclinical ADME lies within ICH S6(R1), where the focus is the safety evaluation of biotechnology derived pharmaceuticals. Clinical ADME evaluations are currently covered in guidance document such as "Guidelines on the Clinical Investigation of the Pharmacokinetics of Therapeutic Proteins (EMA, 2007)".

Many publications describe individual or, in some instances, combined ADME processes that can be assessed for a specific class of TPs, e.g. monoclonal antibodies (Lee, 2013). It appears therefore, that academic and industry groups engage in characterizing the ADME properties of TPs, but the context may not appear to directly relate to regulatory submissions. We therefore conducted an industry-wide survey to gauge current ADME practices for TPs. We also analyzed BLA applications to further understand submitted/accepted ADME data packages beyond the published guidance documents. It should be noted the scope of this review and survey was focussed on TPs, as exemplified in Fig. 1. Other modalities such peptides, oligonucleotides, vaccines, gene and cell therapy were deemed out of the scope this publication. In addition, an industry white paper on ADME of ADCs was published earlier (Kraynov et al., 2016) and hence ADCs were not incorporated in this activity. The focus here is to share the results of the survey, as well as provide future perspectives on the positioning of ADME studies for TPs in the drug discovery and development workflow.

\section{Industry Survey and BLA Reviews}

To understand the current ADME practices of TPs, an industry wide survey was conducted with IQ member companies between March and May 2021. A total of 26 major questions along with 13 sub-questions were prepared by the IQ working group, broadly covering absorption, distribution, metabolism, excretion, and regulatory strategy of TPs. Only one collated response was accepted from each representative company. Briefly, the questionnaire started with requesting the information on the route of administration (intravenous, 
subcutaneous and others) of TPs. The questions around absorption were focused on studies conducted to understand the mechanism of absorption and in vivo models used to investigate pharmacokinetics of TPs. Distribution questions gathered information on the studies conducted to understand tissue distribution of TPs in preclinical species/humans and the utility of the data in PK/PD modelling. The metabolism/biotransformation questions asked if participating companies perform biotransformation studies and about the utility of biotransformation data in characterizing the disposition of TPs. Although excretion is usually not characterized for TPs, the questions were included to know more about the general practices across the industry. The last section assessed if the participating companies had any specific regulatory strategies around ADME characterization, regulatory interaction experience, and the requirement for additional guidance. The questions included were either multiple choice answers, dichotomous (e.g. yes/no) or with open responses. The data collected from the survey were blinded and processed by the IQ secretariate prior to distribution to the working group. The survey responses were analyzed by the working group to derive inferences. An overall picture of ADME assessments of TPs is shown in Fig. 2. Considering the diversity of modalities and complexity of ADME processes, the survey didn't attempt to focus on a specific modality within TPs, but rather considered TPs as a broader category. We also reviewed the regulatory submission packages of FDA approved TPs between 2011-2020, focusing on ADME data (Table 1), to understand if such investigations have been used in submission packages (Supplementary Table 2; Supplementary Table 3). Since ADME of ADCs was discussed and reviewed by another IQ working group earlier (Kraynov et al., 2016)(Li et al., 2021), we excluded ADCs in the survey.

\section{Absorption}


Absorption is characterized for new drugs with the intent to ensure the drug achieves the desired systemic circulation or tissue concentration, depending on the routes of administration. Typically, absorption is described as oral absorption from the gastrointestinal system or topical absorption through skin for NCEs. The absorption assessment for TPs can include several routes of administration including subcutaneous, intramuscular, inhalation, or local tissue administration, such as intravitreal administration to the eye. Based on the survey responses, the most frequently used routes of administration of TPs are intravenous (IV, $68 \%$ ), followed by subcutaneous (SC, 23\%) and others (e.g. intravitreal and intra-tumoral, 9\%) (Fig. 3A).

Early in preclinical development, the pharmacokinetics of TPs are evaluated in animals. TPs are often evaluated following a single dose intravenous administration and for many cases this is usually conducted in non-human primates (NHPs) (20 out of 22 responses, 91\%), followed by wild-type mice (16 out of 22 responses, $73 \%$ ), and FcRn transgenic mice (13 out of 22 responses, 59\%). (Fig. 3B). As NHP physiology is similar to human (Glassman et al., 2015), single species allometry from NHP data has been used to successfully predict human PK of monoclonal antibodies and other TPs, in the absence of target mediated drug disposition (TMDD) (Avery et al., 2016). Transgenic mice which express human FcRn have also now been demonstrated to successfully predict linear (i.e. non-TMDD) human PK of monoclonal antibodies (Avery et al., 2016). In addition, other preclinical species such as rats, murine tumor models, and SCID Beige mice may be utilized to investigate absorption, guide molecule design and/or to understand the mechanism of action.

With subcutaneous (SC) administration becoming more common for TPs, especially as it may enhance convenience for patients, a number of groups have tried to assess which preclinical species can be used to predict subcutaneous bioavailability. Richter et al investigated sites of administration in minipigs to evaluate the optimal SC site that translates 
to humans (Richter et al., 2020). A monoclonal antibody was dosed at four SC regions (interscapular area, flank, behind ear and inguinal area) and showed there was a difference in bioavailability from the four sites. The rate of absorption is key in selecting the SC dose site and the observed rate varied across the different minipig dose sites. The bioavailability from the injection behind the ear and interscapular area best correlated with the human PK data. In addition, the thickness of the SC tissue in minipigs should be taken into consideration when conducting PK studies (Richter et al., 2020).

In the human setting, site of injection can, in some instances, affect the bioavailability and achieved concentrations in serum or plasma. Examples such as dulaglutide, a peptide-Fc fusion protein, exemplified no obvious differences in exposure after administration to the abdomen, upper arm or thigh (Geiser et al., 2016). However, for some monoclonal antibodies, statistically significant differences have been observed when comparing injection to the thigh or abdomen (Bittner et al., 2018). Known examples include secukinumab (Bruin et al., 2020), ixekizumab (Talz label), bococizumab (Wang et al., 2017), and mepolizumab ( Ortega et al., 201). For golimumab, statistically non-significant differences were observed (Xu et al., 2010). There are cases of significant differences in terms of exposure based on the site of injection, exemplified by human growth hormone (hGH) (Beshyah et al., 1991). Investigators assessed the absorption following a single SC injection of hGH to the abdomen versus thigh in healthy young adults. The hGH was administered on two distinct occasions, first into the thigh and then into the abdomen on a subsequent visit. Absorption of hGH was significantly higher following SC injection to the abdomen compared to the thigh. It was unclear why this was the case, but showed that absorption assessment should be done, especially with TPs that have narrow therapeutic windows.

Most companies surveyed do not investigate mechanisms of absorption (91\%) for TPs. Among the two companies who did, one conducted bioavailability studies and evaluated in 
vivo structure/function relationships while the other may use techniques such as photoacoustic imaging to study the absorption of peptides and monoclonal antibodies. Additionally, this company also investigated the correlation between bioavailability and biophysical properties, which include hydrophobicity, aggregation potential, non-specific binding potential, charge patches, and binding to FcRn. These types of investigations impacting absorption are likely being conducted during the molecular design and selection stage, as well as lead optimization of a TP in discovery, and not necessarily as part of the development package for a regulatory submission.

Several factors can impact the absorption and PK profiles of TPs in animals and humans. These include bioavailability, anti-drug antibody (ADA) formation, and target meditated drug disposition.

i) Bioavailability: determining the bioavailability from the SC dose site demonstrates that a portion of the TP is absorbed into the blood circulation via the lymphatics or directly into the blood stream and redistributed to the site of action. The bioavailability of monoclonal antibodies can range from 50 to 100\% (Deng et al., 2012). Differences observed can be molecule, physiology and route dependent (Collins et al., 2020). The absorption of antibodies is known to be FcRn dependent, and this may also impact SC bioavailability in humans (Glassman and Balthasar, 2019). There are challenges in developing preclinical models across species in order to accurately predict bioavailability in humans. For some companies, the decision to use the subcutaneous route early in program development means that true bioavailability (i.e. compared to intravenous administration) is not necessary. However, formulation or device modifications during later clinical development will require relative bioavailability assessments. 
ii) Anti-drug antibodies (ADAs): an essential element of the ADME data package is to evaluate the potential for ADA formation to the TP (Chirmule et al., 2012). The administration of TPs to animals and humans may trigger an immunogenic response which may lead to an impact on the exposure, bioavailability and efficacy of the TP. The presence of ADAs may also interfere with the bioanalytical method used to measure the $\mathrm{PK}$ of the $\mathrm{TP}$ and thus requires careful consideration during method development (Thway et al., 2013; Ryman and Meibohm, 2017). Overall, although the incidence of $\mathrm{ADA}$ formation in animals does not always translate to humans, it is important to understand immunogenicity in both animals and humans as part of the TP regulatory submission package, to correctly interpret toxicology study results and ensure human safety and efficacy, respectively.

iii) Target-mediated drug disposition (TMDD): this is a phenomenon whereby the amount and turnover of the target can influence the absorption or pharmacokinetic profile of the TP significantly. This occurs often with TPs because the kinetics of binding, and especially the disassociation rates involved between target and TP, are often much slower than physiological processes governing target behavior. When conducting PK studies with TPs, several dose levels of TP may be needed to fully evaluate the exposure-response relationship in the presence of TMDD, where it may also be important to assess if TMDD can be saturated with high doses. The extent of TMDD may vary between nonclinical species and humans (Glassman et al., 2015), and should be carefully considered. Mechanistic mathematical models can be developed to account for this phenomenon in order to support initial human dose projections.

In summary, for absorption, although the IV route is still the most frequent route of administration, per the IQ survey (Fig. 3A), other routes of administration are being explored 
and utilized for TPs. For more recently approved TPs and TPs under development, SC administration is becoming the desired route across many different therapeutic indications (Turner and Balu-Iyer, 2018; Collins et al., 2020). The absorption and full pharmacokinetic profile of the TP are the foundation for understanding and achieving adequate exposure for therapeutic benefits. Absorption assessment remains a key component to the overall ADME data package for delivery of TPs via non-intravenous routes.

\section{Distribution}

Tissue biodistribution studies contribute to the knowledge about the disposition of TPs, which is critical to understand efficacy (site of action/target tissue) and safety (off target tissue) from lead selection to clinical development. Physicochemical properties such as molecular size, isoelectric point, glycosylation, and target binding affinity play a role in distribution of TPs and hence these factors should be carefully considered for selection of TP for development (Tabrizi et al., 2010).

As shown in Table 1, only 20 out of the 91 BLAs (22\%) submitted from 2011 to 2020 contained preclinical distribution data as measured in mice, rats, guinea pigs or monkeys. Interestingly, the majority of the companies in the survey (77\%, 17 out of 22 , Fig. 2) indicated that they analyze biodistribution to target tissues, for instance by measuring target tissue drug concentrations in preclinical species (Fig. 4). The survey also revealed that biodistribution of TPs to target tissue in human subjects is analyzed by $50 \%$ of the companies (Fig. 4). The discrepancy between distribution data presented in BLAs (22\%) and the survey (77\%) might suggest that many preclinical distribution studies are exploratory in nature and are not being submitted in the BLAs. However, the survey results also partly indicate a trend that companies are increasing their efforts to understand the distribution processes of TPs. 
Many analytical techniques are available to study biodistribution of TPs. Survey results indicated that the tools used to evaluate preclinical and human biodistribution to target tissues are similar (Table 2). Examples of such techniques and methods include Ligand Binding Assays (LBAs), LC-MS/MS, and imaging technologies utilizing fluorescence or radiolabeling approaches. For instance, Quantitative Whole Body Autoradiography (QWBA) methods with ${ }^{3} \mathrm{H}$-labeled proteins can give valuable quantitative insights into the whole body distribution in rodents or NHPs. Other radiotracers such as ${ }^{111} \mathrm{In},{ }^{125} \mathrm{I},{ }^{89} \mathrm{Zr}$ are frequently employed, particularly for in-life imaging (Conner et al., 2020). Fc-containing TPs are most commonly labeled with ${ }^{125} \mathrm{I}$, which might be mainly due to early development of labeling chemistry involving the attachment of iodine atoms to tyrosine residues in proteins (Cohen et al., 1956). Recent advancement of labeling chemistry and imaging technologies have provided more opportunities to characterize biodistribution of therapeutic drugs. While traditional QWBA methods require an animal per time point, Positron Emission Tomography (PET) or Single-Photon Emission Computed Tomography (SPECT) techniques enable image collection from live subjects at multiple time points, finding applicability in not only nonclinical species but also in humans (Gomes et al., 2011). In addition, PET combined with CT gives robust contrast and spatial resolution.

Generally, the need for sampling of target tissue(s) in a clinical setting is only judged on a case-by-case basis depending on accessibility, volumes of biopsy etc. Understanding the TP concentrations in target tissues in humans is critical to predict optimal dose and dosing regimen design. Examples of sampling techniques include open flow microperfusion or simply withdrawal of blister fluid in case of TPs developed for psoriasis or withdrawal of synovial fluid in rheumatoid arthritis patients (Choy and Panayi, 2001; Dragatin et al., 2016). Furthermore, CSF sampling can be of interest for evaluation of CNS distribution. As an example, CSF-to-serum ratio of less than $0.5 \%$ for five independent antibodies in rats and 
NHPs were measured and agree well with CSF-to-total serum IgG ratios of $0.1-0.2 \%$ in healthy humans as reported by several groups (Naessens et al., 2018; Wang et al., 2018; Brys et al., 2019).

In the case of conducting biodistribution studies with TPs, the contribution of TMDD should be clearly distinguished from nonspecific distribution mechanisms and quantified accordingly. Some recommendations can be made in this respect:

i) Nonspecific biodistribution can be studied by in vitro for example, assessing interactions with HEK cells (Datta-Mannan et al., 2015), as well as in animals that do not express the target of the TP or the TP is not cross-reactive with the target expressed in that species ii) TMDD can be very well evaluated with single and multiple dose PK in nonclinical species (if TP has similar binding properties). In nonclinical species, dose - exposure - response relationships to determine regions of nonlinearity can be correlated with biodistribution results (i.e., receptor occupancy by flow cytometry).

iii) A good understanding of target expression in tissues together with robust multicompartmental modeling might be pivotal for a robust understanding of PK/PD relationships and translation to human predictions. The survey results indicated most companies (82\%) use distribution results in translational PK/PD modelling for efficacy and safety predictions. This nicely demonstrates the increasing role of modelling and simulation strategies that utilize TP biodistribution data. More details on the utility of biodistribution data in PK/PD modeling is presented in the companion manuscript of this working group (Ball et al., 2022).

There are numerous examples of preclinical and clinical investigation of biodistribution of TPs. Some enable human dose setting, whilst others eventually lead to USPI (United States product insert) and/or SmPC (summary of product characteristics) labeling. Three examples are given below. 
i. Biodistribution of various recombinant antibody platforms was assessed with respect to tumor accumulation and kinetics in xenograft mice models (Schneider et al., 2009). Four different antibody based therapeutic platforms, mAb, diabody, scFv, and novel miniantibody were labeled with PET tracers, and evaluated in prostate cancer cell xenograft mouse studies. The authors conclude that larger antibody frameworks (IgG and miniantibody) gave higher tumor uptake levels than smaller frameworks (diabody and scFv). These larger frameworks may be more suitable for radioimmunotherapy applications while the smaller ones may be suitable for radiodiagnostic applications.

ii. Active transplacental transport of $\operatorname{IgG}$ from mother to infant is mediated by FcRn in the placenta, mainly during the second and third trimesters of pregnancy (Pentsuk and van der Laan, 2009). As a result, various alternative formats have been assessed to avoid significant exposure of the drug to the developing fetus. One example is the PEGylated, Fc-free anti-TNF Fab called certolizumab pegol $\left(\right.$ CIMZIA $\left.^{\circledR}\right)$, approved for the treatment of various autoimmune diseases. Due to the lack of an IgG Fc region, certolizumab pegol does not bind FcRn and is consequently not expected to undergo transfer across the placenta. This was confirmed in a clinical study in which sixteen pregnant women were assessed at term for transfer to their babies. None to minimal exposure of certolizumab pegol in babies was observed, suggesting lack of placental transfer during pregnancy (Mariette et al., 2018). A more recent example explored temporal and quantitative placental transfer of humanized $\operatorname{IgG} 2 \Delta$ a format in cynomolgus monkeys (Catlin et al., 2020). Such efforts with more novel formats would also improve the assessment of teratogenic potential of TPs and enhance our ability to extrapolate relevance of these data to humans.

iii. Based on recent review of nineteen selected $\mathrm{mAb}$ therapeutics, distribution to breast milk was typically at low concentrations and tended to peak within 48 hours, although 
maximum levels could occur up to 14 days from infusion (LaHue et al., 2020). The relative infant dose, a metric comparing the infant with maternal drug dose $(<10 \%$ is generally considered safe), was evaluated for three mAbs and was $<5.3 \%$. Importantly, a total of 368 infants were followed for $\geq 6$ months after exposure to breast milk of mothers treated with mAbs; none experienced reported developmental delay or serious infections. In conclusion, the data confirmed low mAb drug transfer to breast milk, but further studies are needed (keeping in mind that data were largely derived from case reports), especially with regard to longer-term effects on infant immunity and childhood development.

In summary, a robust knowledge of biodistribution properties of TPs is beneficial for enabling reliable predictions of in vivo PK and target tissue distribution. Characterization of the tissue exposure to TPs can be challenging, but efforts in this respect at the early stages of development can be helpful to reveal mechanisms governing TP pharmacology and/or toxicity. Continued research in the field of TP biodistribution is strongly encouraged. Specific areas of focus include deeper understanding of physico-chemical properties and their relationship to distribution of TPs, target expression profiles in preclinical species and human, and continued improvements in biosampling in the clinical setting.

\section{Metabolism}

There is increasing interest in gaining insights into the metabolism of TPs. Depending on the intended purpose, specific studies on biotransformation, a general terminology used in this paper to describe metabolism, catabolism and other modifications and degradations, may be considered at various stages of drug discovery and development.

Biotransformation is generally the process of conversion of xenobiotics, i.e., non-endogenous compounds, to products that are suitable for elimination from the body. Understanding of the biotransformation of NCEs is essential, as it could lead to the formation of pharmacologically 
or toxicologically active metabolites or lead to drug-drug interaction potential which often trigger efficacy and/or safety concerns. Therefore, biotransformation of small molecules is continuously monitored from preclinical studies through clinical development.

Biotransformation of TPs includes hydrolysis of amide bonds in the protein backbone, metabolism of amino acid side chains, and other post translational modifications (PTMs) such as glycation. Biotransformation may alter the PK, ADME and efficacy, but rarely increase the toxicity of the TPs. Hydrolysis of amide bonds by proteases in vivo is a major clearance pathway of TPs. Biotransformation products (BPs) from proteolysis of TPs may lose their biological activity since the target binding site may be cleaved or geometrically changed. In cases of hydrolysis occurring on one or a few amino acids at the C- or Nterminus of a TP, BPs may still retain partial or full pharmacokinetic properties and/or pharmacological activities. Proteolytic reactions are expected to occur in lysosomes and endosomes after endocytosis, in the proximal tubule post-glomerular filtration, and in the circulatory system or extracellular fluid by proteases. For example, therapeutic mAbs are mainly catabolized in lysosomes inside cells by resident proteases. Some TPs, particularly the recombinant endogenous peptide hormones such as GLP-1 and GIP, are very sensitive to the proteases and peptidases in the circulatory system (Mentlein et al., 1993; Werle and BernkopSchnurch, 2006). Their plasma half-life can be extremely short in vivo due to both proteolysis in the blood and glomerular filtration. To mitigate fast clearance by glomerular filtration, recombinant endogenous proteins, peptide hormones, or their analogues can be fused or conjugated to large proteins such as mAb (Rangwala et al., 2019), Fc domains (Glaesner et al., 2010; Hall et al., 2010; Hecht et al., 2012; Lee and Lee, 2017), human albumin (Baggio et al., 2004; Matthews et al., 2008) and polyethylene glycol (PEG) (Jevsevar et al., 2010) to increase the molecular sizes. However, the protein or peptide moiety in a fusion or conjugate TP can still be susceptible to cleavages induced by proteases and peptidases. To minimize 
potential proteolysis of fusion or conjugate TPs, different strategies can be used (Walles et al., 2021). Integrated analytical strategies, often involving ligand binding assay (LBA) and hybrid immunoaffinity LC-MS platforms, are being adopted gradually over time to gain better understanding of biotransformation of TPs (Kaur et al., 2020).

Biotransformation of amino acid side chain such as oxidation, deamidation, and isomerization, as well as other PTMs like glycation may impact critical properties of the molecule. For example, oxidation of key methionine residues on human IgG weakens the FcRn or Fc- $\gamma$ receptor binding affinity, which can negatively affect the PK and/or effector functions (Gao et al., 2015). Similarly, modification of the amino acid side chain in the CDR (complementarity- determining region) region of a mAb may ablate the antigen binding affinity and result in the decreased pharmacokinetics and activities (Dashivets et al., 2016; Menke-van der Houven van Oordt et al., 2016) (Bults et al., 2016). Glycation of serum albumin is a known modification that occurs in circulation, especially in disease states such as diabetes. Recent studies have shown that the interaction of glycated albumin, particularly at position K525, leads to a reduction in affinity for FcRn (Leblanc et al., 2019). Molecules involving albumin fusions may require a deeper characterization to understand the PK impact in certain clinical populations. Some evidence suggested possible differences in biotransformation across certain patients (Liu et al., 2018).

Traditionally, biotransformation studies for TPs are not required for regulatory filing. In general, unlike NCEs, safety risk due to biotransformation of the TP is believed to be minimal. The risk for inhibition or induction of metabolizing enzymes by the BPs is considered relatively low and thus not monitored in general, unless the mechanism of action is known to impact CYP expression, e.g. IL-6 (Schmitt et al., 2011). Among the regulatory submission packages analyzed, for the majority of the approved biotherapeutics, no biotransformation data was included. 
In line with the observation that biotransformation is in general not conducted in regulatory filings of TPs, specific guidance from the health authorities on this subject has been limited. In this regard, the ICH Topic S6 guideline and the European Medicines Agency guideline on the clinical investigation of the pharmacokinetics of TPs are often cited. Under the ICH Topic S6 guideline issued in 1998, there was no expectation in conducting conventional preclinical metabolism studies. Nevertheless, stability testing in the matrix used for bioanalysis (e.g., plasma or serum) is recommended to understand the behavior of the biopharmaceutical and the possible influence of binding proteins to the pharmacodynamic effect. The more recent European Medicines Agency clinical guidance for pharmacokinetics (PK) of TPs suggests a case-by-case consideration for in vitro metabolism studies (EMA, 2007), recognizing that it could be relevant for certain protein drugs, particularly conjugated and altered protein sequence drugs, where the cleavage products could still contribute to the overall pharmacological activity.

Although biotransformation of the TP is typically not required for regulatory submission, interestingly, the survey results indicate that there is an almost even-split among the responding companies - 54\% perform metabolism/biotransformation studies while $46 \%$ do not (Fig. 2). It is worth noting that these numbers may largely depend on the drug modalities in the pipeline of each company. Typically, biotransformation is not performed for monoclonal antibodies while specific studies may be conducted for fusion proteins, protein/peptide conjugates, proteins with in vivo modification liability or unexpected PK behaviors. It is believed that the biotransformation assessments are most likely conducted in the discovery and early development phases to help optimize and select the TP. A detailed overview of biotransformation studies to address the liabilities of TPs can be found in the companion manuscript (Walles et al., 2021). 
The majority of the survey companies (82\%) have not established IVIVC (in vitro-in vivo correlation) with regards to metabolism of TPs. However, certain reaction-driven PTMs in buffer (deamidation or isomerization) may be used to predict in vivo liability and serum incubation to predict in vivo stability. Most companies (77\%) do not perform cross species metabolite profiling, which is typically done for small molecules. Analytical platforms used for biotransformation evaluation so far are primarily mass spectrometry and LBA. The majority of the companies (86\%) do not use radiolabeled TPs to elucidate biotransformation pathways. Quantification of active metabolites/catabolites is not usually pursued. In general, there has not been a well-established strategy in studying biotransformation yet, with studies being conducted on a fit-for-purpose basis. Depending on the individual company and the drug modalities in its pipeline, the biotransformation data could be used for gaining insights into the areas of clearance mechanism, PK/PD disconnects, safety, regulatory and others, as depicted in Fig. 5.

\section{Excretion}

For smaller MW proteins $(\sim<60 \mathrm{kDa})$, kidney is the primary elimination organ with high levels of renal filtration and subsequent degradation after proximal tubule reabsorption (Maack et al., 1979). For instance, FGF21 is a small protein with a MW of $\sim 19 \mathrm{kDa}$ used in the treatment of type 2 diabetes. It has a very short half-life in rodents and NHPs, which is partially attributed to the renal excretion (Hager et al., 2013). Interleukin 2 (IL-2) is a cytokine with $15.6 \mathrm{kDa}$ in MW which plays an important role in the immune system. The terminal half-life of recombinant IL-2 was 85 minutes in human with the renal filtration as the major clearance pathway (Konrad et al., 1990). Interferon- $\alpha(\mathrm{IFN}-\alpha)$ is a group of cytokines with MWs ranging from 17.5 to $23 \mathrm{kDa}$, which are also cleared mainly by renal filtration (Wills, 1990). 
Several strategies to improve the pharmacokinetic properties of lower molecular weight TPs, specifically to maximize the half-life in the body, have been described the metabolism section. Polyethylene glycol (PEG) polymers are perceived to be nontoxic, nonimmunogenic, non-antigenic, highly soluble in water, and have been approved by agencies for use in humans when chemically conjugated to low molecular weight TPs e.g. certolizumab pegol $\left(\operatorname{Cimzia}^{\circledR}\right)$ or pegvisomant $\left(\right.$ Somavert $\left.^{\circledR}\right)$. However, elimination of PEGylated proteins is not yet well understood. Data for certolizumab pegol, a Fab conjugated with a 40kDa PEG moiety, has shown that its PEG moiety is almost entirely excreted unchanged in the urine once cleaved from the Fab (Nesbitt et al., 2007).

The survey sought responses about studies conducted to understand excretion of TPs. The majority of companies do not determine elimination routes of TPs either in preclinical species (82\%) (Fig. 2) or in humans (91\%). These studies are generally performed for fusion and PEGylated proteins (e.g. cytokines, growth factors), nanobodies, peptides and ADCs.

Reviewed data from BLA submissions suggests that elimination studies, when conducted, use rodents or NHPs. For higher MW TPs like antibodies, the renal excretion plays a minimum role in their clearance as their size is too large to be filtered by glomerulus. However, higher MW proteins can be excreted by the kidney under certain disease conditions such as nephrotic syndrome and glomerular proteinuria (Waldmann et al., 1972). Similar to the renal excretion, biliary excretion plays a very minor role to the elimination of large MW proteins such as IgGs (Thomas and Balthasar, 2019). Instead, both active (receptor-mediated endocytosis) and passive (fluid-phase endocytosis) mechanisms of protein transport from the vascular endothelium to the underlying tissue and subsequent intracellular degradation are typically major elimination pathways for large proteins (Wang et al., 2008).

\section{Regulatory Strategy}


With regards to regulatory strategies for TPs, most companies (86\%) do not have an internal regulatory strategy specifically for ADME of TPs (Fig. 6A). Regulatory interactions from most companies (73\%) did not lead to any additional ADME-focused studies for TPs (Fig. 6B). Interactions that led to ADME studies for TPs were primarily related to drug-drug interactions (3 companies). Based on the survey results, the expectation on the need for further regulatory guidance on ADME of TPs, on a scale of 1 (none needed) to 10 (yes, absolutely need more), the median value of the response was 4.5 and the mean was 4.2 (Fig. 6C).

\section{Conclusions and future perspectives}

Over the last couple of decades, there has been a significant rise in TP drug discovery and development. Due to major differences in physicochemical properties of TPs compared to small molecule drugs, an orthogonal approach and mindset has been taken in terms of ADME characterization and regulatory requirements. The goal of our exercise was to evaluate the nature of ADME studies used in regulatory BLA submissions, compare this to current ADME practices in IQ member companies and provide recommendations for conducting ADME studies of TPs. A disparity exists between activities conducted by companies in TP programs and what has been submitted as part of regulatory packages. As it stands at the moment, regulatory guidance or requirements for ADME studies of TPs is limited. This would therefore suggest that most companies are exploring various ADME characteristics of their TPs in order to optimize their molecules, made via protein engineering or formulation early during the discovery/preclinical phases of drug development. As the diversity of protein engineering continues to extend further away from classical monoclonal antibodies, more endeavors in the discovery phase will no doubt continue to be needed in order to characterize the performance of novel modalities. The need for a more-NCE oriented thinking is still necessary once TPs are conjugated to chemical entities, such as toxins or PEG, or non-natural 
amino acids. We would also like to provide future perspectives for ADME studies for TPs, considering specifically the needs for regulatory submission.

In cases where TPs are not administered intravenously, absorption should continue to be assessed in order to understand the PK in nonclinical species and the translatability to humans. With respect to subcutaneous administration, sites of injection as well as bioavailability have been shown to influence TP's exposure, and this may need continued evaluation, especially with molecules that exhibit narrow therapeutic indices. Local administration of TPs, e.g., to the eye, lung or cartilage, will still require an assessment of absorption into the systemic circulation to enable risk assessment of wider exposure in the body, albeit usually at a very low concentration. PK should be continued to be assessed in NHPs and other preclinical species as appropriate for the program.

With advancement of sampling and detection technologies, there have been increased efforts to understand biodistribution of TPs. Although there is no requirement for regulatory submissions, it is anticipated that more question based fit-for-purpose studies are to be conducted and emphases will certainly be on incorporating biodistribution data in translational PK/PD modeling for a better understanding of systemic and target exposureresponse in terms of efficacy and safety.

For TPs in general the need to perform biotransformation studies lies mainly with discovery/preclinical stages to facilitate the selection and optimisation of a likely candidate molecule. With this in mind, it is not envisaged at this time, that regulatory metabolism studies are required. However, biotransformation assessments may be needed for modalities such as fusion and PEGylated proteins, and ADCs.

The impact of excretion on PK or clearance is negligible for TPs with large MW (> 60 $\mathrm{kDa})$. For TPs with small MW $(<\sim 60 \mathrm{kDa})$, the renal excretion may play a significant role in 
their clearance. Therefore, both the size of the TPs and any special populations need to be taken into consideration to determine the elimination pathways.

Upon review of submitted BLAs over the last 10 years, it is apparent that most submissions contain limited ADME data (Supplementary Table 2; Supplementary Table 3)). The main exception to this is information on absorption, likely due to the increasing desire for patient convenience facilitated by subcutaneous administration. Unlike NCEs, TPs typically require limited ADME assessments when considering patient safety. Physiological processes determining the disposition and elimination of TPs are broadly consistent across species (except for TMDD), further underlining the accepted level of detail provided in ADME packages.

The desire for greater regulatory guidance is moderate according to the survey, suggesting that there is a need for some further clarity in guidance, whilst recognizing that due to the significant diversity of TPs, as well as lower risk of off-target or idiopathic safety issues, overarching guidance is likely not critically needed. A case-by-case approach examining the key aspects and associated risks of a given TP, alongside robust understanding of target biology and how this impacts the ADME behaviours of the TP, should continue to be considered. Impact of immunogenicity of TPs on drug exposure in both preclinical and clinical settings, alongside bioavailability evaluations where needed, should also remain crucial aspects in regulatory submissions. Continued dialogue between agencies and the industry will also be crucial to ensure this approach is reviewed and evolved as needed.

\section{Acknowledgements}

The authors thank the companies that participated in the survey. The authors also thank International Consortium for Innovation and Quality (IQ) secretariat who helped in sending 
the survey and compiling the responses. Lastly, authors would like to thank IQ TALG for support of this work.

\section{Authorship Contribution:}

All authors participated in research design, performed data analysis, and wrote or contributed to the writing of the manuscript.

\section{References}

Avery LB, Wang M, Kavosi MS, Joyce A, Kurz JC, Fan YY, Dowty ME, Zhang M, Zhang Y, Cheng A, Hua F, Jones HM, Neubert H, Polzer RJ, and O'Hara DM (2016) Utility of a human FcRn transgenic mouse model in drug discovery for early assessment and prediction of human pharmacokinetics of monoclonal antibodies. MAbs 8:1064-1078.

Baggio LL, Huang Q, Brown TJ, and Drucker DJ (2004) A Recombinant Human Glucagon-Like Peptide (GLP)-1-Albumin Protein (Albugon) Mimics Peptidergic Activation of GLP-1 ReceptorDependent Pathways Coupled With Satiety, Gastrointestinal Motility, and Glucose Homeostasis. Diabetes 53:2492-2500.

Ball K, Bruin G, Escandon E, Brady K, Funk C, Pereira JN, Yang T-Y, and Yu H (2022) Characterizing the Pharmacokinetics and Biodistribution of Therapeutic Proteins: An Industry Perspective. Drug Metab Dispos (in preparation).

Beshyah SA, Anyaoku V, Niththyananthan R, Sharp P, and Johnston DG (1991) The effect of subcutaneous injection site on absorption of human growth hormone: abdomen versus thigh. Clin Endocrinol (Oxf) 35:409-412.

Bittner B, Richter W, and Schmidt J (2018) Subcutaneous Administration of Biotherapeutics: An Overview of Current Challenges and Opportunities. BioDrugs 32:425-440.

Brady K and Webster R (2012) Disposition of biologics. Adv Pharmacol 63:257-277.

Bruin G, Hockey HP, La Stella P, Sigurgeirsson B, Fu R, Patekar M, Charef P, Woessner R, and Boutouyrie-Dumont B (2020) Comparison of pharmacokinetics, safety and tolerability of secukinumab administered subcutaneously using different delivery systems in healthy volunteers and in psoriasis patients. Br J Clin Pharmacol 86:338-351.

Brys $M$, Fanning L, Hung S, Ellenbogen A, Penner N, Yang M, Welch M, Koenig E, David E, Fox T, Makh S, Aldred J, Goodman I, Pepinsky B, Liu Y, Graham D, Weihofen A, and Cedarbaum JM (2019) Randomized phase I clinical trial of anti-alpha-synuclein antibody BllB054. Mov Disord 34:1154-1163.

Bults P, Bischoff R, Bakker H, Gietema JA, and van de Merbel NC (2016) LC-MS/MS-Based Monitoring of In Vivo Protein Biotransformation: Quantitative Determination of Trastuzumab and Its Deamidation Products in Human Plasma. Anal Chem 88:1871-1877.

Catlin NR, Mitchell AZ, Potchoiba MJ, O'Hara DM, Wang M, Zhang M, Weinbauer GF, and Bowman CJ (2020) Placental transfer of (125) iodinated humanized immunoglobulin G2Deltaa in the cynomolgus monkey. Birth Defects Res 112:105-117.

Chirmule N, Jawa V, and Meibohm B (2012) Immunogenicity to therapeutic proteins: impact on PK/PD and efficacy. The AAPS journal 14:296-302.

Choy EH and Panayi GS (2001) Cytokine pathways and joint inflammation in rheumatoid arthritis. N Engl J Med 344:907-916. 
Cohen S, Holloway RC, Matthews C, and McFarlane AS (1956) Distribution and elimination of $131 \mathrm{I}-$ and 14C-labelled plasma proteins in the rabbit. Biochem J 62:143-154.

Collins DS, Sanchez-Felix M, Badkar AV, and Mrsny R (2020) Accelerating the development of novel technologies and tools for the subcutaneous delivery of biotherapeutics. J Control Release 321:475-482.

Conner KP, Devanaboyina SC, Thomas VA, and Rock DA (2020) The biodistribution of therapeutic proteins: Mechanism, implications for pharmacokinetics, and methods of evaluation. Pharmacol Ther 212:107574.

Dashivets T, Stracke J, Dengl S, Knaupp A, Pollmann J, Buchner J, and Schlothauer T (2016) Oxidation in the complementarity-determining regions differentially influences the properties of therapeutic antibodies. MAbs 8:1525-1535.

Datta-Mannan A, Thangaraju A, Leung D, Tang Y, Witcher DR, Lu J, and Wroblewski VJ (2015) Balancing charge in the complementarity-determining regions of humanized mAbs without affecting $\mathrm{pl}$ reduces non-specific binding and improves the pharmacokinetics. MAbs 7:483493.

Deng R, Jin F, Prabhu S, and lyer S (2012) Monoclonal antibodies: what are the pharmacokinetic and pharmacodynamic considerations for drug development? Expert Opin Drug Metab Toxicol 8:141-160.

Doogue MP and Polasek TM (2013) The ABCD of clinical pharmacokinetics. Ther Adv Drug Saf 4:5-7.

Dragatin C, Polus F, Bodenlenz M, Calonder C, Aigner B, Tiffner KI, Mader JK, Ratzer M, Woessner R, Pieber TR, Cheng Y, Loesche C, Sinner F, and Bruin G (2016) Secukinumab distributes into dermal interstitial fluid of psoriasis patients as demonstrated by open flow microperfusion. Exp Dermatol 25:157-159.

EMA (2007) Guideline on the Clinical Investigation of the Pharmacokinetics of Therapeutic Proteins.

Gao X, Ji JA, Veeravalli K, Wang YJ, Zhang T, McGreevy W, Zheng K, Kelley RF, Laird MW, Liu J, and Cromwell M (2015) Effect of individual Fc methionine oxidation on FcRn binding: Met252 oxidation impairs FcRn binding more profoundly than Met428 oxidation. J Pharm Sci 104:368-377.

Geiser JS, Heathman MA, Cui X, Martin J, Loghin C, Chien JY, and de la Pena A (2016) Clinical Pharmacokinetics of Dulaglutide in Patients with Type 2 Diabetes: Analyses of Data from Clinical Trials. Clin Pharmacokinet 55:625-634.

Glaesner W, Mark Vick A, Millican R, Ellis B, Tschang S-H, Tian Y, Bokvist K, Brenner M, Koester A, Porksen N, Etgen G, and Bumol T (2010) Engineering and characterization of the long-acting glucagon-like peptide-1 analogue LY2189265, an Fc fusion protein. Diabetes/Metabolism Research and Reviews 26:287-296.

Glassman PM and Balthasar JP (2019) Physiologically-based modeling of monoclonal antibody pharmacokinetics in drug discovery and development. Drug Metab Pharmacokinet 34:3-13.

Glassman PM, Chen Y, and Balthasar JP (2015) Scale-up of a physiologically-based pharmacokinetic model to predict the disposition of monoclonal antibodies in monkeys. J Pharmacokinet Pharmacodyn 42:527-540.

Gomes CM, Abrunhosa AJ, Ramos P, and Pauwels EK (2011) Molecular imaging with SPECT as a tool for drug development. Adv Drug Deliv Rev 63:547-554.

Hager T, Spahr C, Xu J, Salimi-Moosavi H, and Hall M (2013) Differential Enzyme-Linked Immunosorbent Assay and Ligand-Binding Mass Spectrometry for Analysis of Biotransformation of Protein Therapeutics: Application to Various FGF21 Modalities. Analytical Chemistry 85:2731-2738.

Hall MP, Gegg C, Walker K, Spahr C, Ortiz R, Patel V, Yu S, Zhang L, Lu H, DeSilva B, and Lee JW (2010) Ligand-Binding Mass Spectrometry to Study Biotransformation of Fusion Protein Drugs and Guide Immunoassay Development: Strategic Approach and Application to Peptibodies Targeting the Thrombopoietin Receptor. The AAPS journal 12:576-585. 
Hamuro LL and Kishnani NS (2012) Metabolism of biologics: biotherapeutic proteins. Bioanalysis 4:189-195.

Hecht R, Li Y-S, Sun J, Belouski E, Hall M, Hager T, Yie J, Wang W, Winters D, Smith S, Spahr C, Tam L$T$, Shen Z, Stanislaus S, Chinookoswong N, Lau Y, Sickmier A, Michaels ML, Boone T, Véniant MM, and Xu J (2012) Rationale-Based Engineering of a Potent Long-Acting FGF21 Analog for the Treatment of Type 2 Diabetes. PLOS ONE 7:e49345.

Jevsevar S, Kunstelj M, and Porekar VG (2010) PEGylation of therapeutic proteins. Biotechnol J 5:113128.

Kaur S, Bateman KP, Glick J, Jairaj M, J FK, Sydor J, and Zeng J (2020) IQ consortium perspective: complementary LBA and LC-MS in protein therapeutics bioanalysis and biotransformation assessment. Bioanalysis 12:257-270.

Konrad MW, Hemstreet G, Hersh EM, Mansell PW, Mertelsmann R, Kolitz JE, and Bradley EC (1990) Pharmacokinetics of recombinant interleukin 2 in humans. Cancer Res 50:2009-2017.

Kraynov E, Kamath AV, Walles M, Tarcsa E, Deslandes A, Iyer RA, Datta-Mannan A, Sriraman P, Bairlein M, Yang JJ, Barfield M, Xiao G, Escandon E, Wang W, Rock DA, Chemuturi NV, and Moore DJ (2016) Current Approaches for Absorption, Distribution, Metabolism, and Excretion Characterization of Antibody-Drug Conjugates: An Industry White Paper. Drug Metab Dispos 44:617-623.

LaHue SC, Anderson A, Krysko KM, Rutatangwa A, Dorsey MJ, Hale T, Mahadevan U, Rogers EE, Rosenstein MG, and Bove R (2020) Transfer of monoclonal antibodies into breastmilk in neurologic and non-neurologic diseases. Neurol Neuroimmunol Neuroinflamm 7, e-769.

Leblanc Y, Berger M, Seifert A, Bihoreau N, and Chevreux G (2019) Human serum albumin presents isoform variants with altered neonatal Fc receptor interactions. Protein Sci 28:1982-1992.

Lee JW (2013) ADME of monoclonal antibody biotherapeutics: knowledge gaps and emerging tools. Bioanalysis 5:2003-2014.

Lee $S$ and Lee DY (2017) Glucagon-like peptide-1 and glucagon-like peptide-1 receptor agonists in the treatment of type 2 diabetes. Ann Pediatr Endocrinol Metab 22:15-26.

Li C, Menon R, Walles M, Singh R, Upreti VV, Brackman D, Lee AJ, Endres CJ, Kumar S, Zhang D, Barletta F, Suri A, Haninzl D, Liao KH, Lalovic B, Beaumont M, Zuo P, Mayer AP, and Wei D (2021) Risk-Based Pharmacokinetic and Drug-Drug Interaction Characterization of AntibodyDrug Conjugates in Oncology Clinical Development: An IQ Consortium Perspective. Clin Pharmacol Ther (Published online).

Liu L, Xu K, Li J, Maia M, Mathieu M, Elliott R, Yang J, Nijem I, and Kaur S (2018) Optimizing hybrid LCMS/MS binding conditions is critical: impact of biotransformation on quantification of trastuzumab. Bioanalysis 10: 1819-1831.

Maack T, Johnson V, Kau ST, Figueiredo J, and Sigulem D (1979) Renal filtration, transport, and metabolism of low-molecular-weight proteins: a review. Kidney Int 16:251-270.

Mariette X, Forger F, Abraham B, Flynn AD, Molto A, Flipo RM, van Tubergen A, Shaughnessy L, Simpson J, Teil M, Helmer E, Wang M, and Chakravarty EF (2018) Lack of placental transfer of certolizumab pegol during pregnancy: results from CRIB, a prospective, postmarketing, pharmacokinetic study. Ann Rheum Dis 77:228-233.

Matthews JE, Stewart MW, De Boever EH, Dobbins RL, Hodge RJ, Walker SE, Holland MC, Bush MA, and Albiglutide Study G (2008) Pharmacodynamics, pharmacokinetics, safety, and tolerability of albiglutide, a long-acting glucagon-like peptide-1 mimetic, in patients with type 2 diabetes. J Clin Endocrinol Metab 93:4810-4817.

Menke-van der Houven van Oordt CW, Gomez-Roca C, van Herpen C, Coveler AL, Mahalingam D, Verheul HM, van der Graaf WT, Christen R, Ruttinger D, Weigand S, Cannarile MA, Heil F, Brewster M, Walz AC, Nayak TK, Guarin E, Meresse V, and Le Tourneau C (2016) First-inhuman phase I clinical trial of RG7356, an anti-CD44 humanized antibody, in patients with advanced, CD44-expressing solid tumors. Oncotarget 7:80046-80058. 
Mentlein R, Gallwitz B, and Schmidt WE (1993) Dipeptidyl-peptidase IV hydrolyses gastric inhibitory polypeptide, glucagon-like peptide-1(7-36)amide, peptide histidine methionine and is responsible for their degradation in human serum. Eur J Biochem 214:829-835.

Naessens DMP, de Vos J, VanBavel E, and Bakker E (2018) Blood-brain and blood-cerebrospinal fluid barrier permeability in spontaneously hypertensive rats. Fluids Barriers CNS 15:26.

Nesbitt A, Fossati G, Bergin M, Stephens P, Stephens S, Foulkes R, Brown D, Robinson M, and Bourne $T$ (2007) Mechanism of action of certolizumab pegol (CDP870): in vitro comparison with other anti-tumor necrosis factor alpha agents. Inflamm Bowel Dis 13:1323-1332.

Ortega H, Yancey S, and Cozens S (2014) Pharmacokinetics and absolute bioavailability of mepolizumab following administration at subcutaneous and intramuscular sites. Clin Pharmacol Drug Dev 3:57-62.

Pentsuk N and van der Laan JW (2009) An interspecies comparison of placental antibody transfer: new insights into developmental toxicity testing of monoclonal antibodies. Birth Defects Res B Dev Reprod Toxicol 86:328-344.

Rangwala SM, D'Aquino K, Zhang Y-M, Bader L, Edwards W, Zheng S, Eckardt A, Lacombe A, Pick R, Moreno V, Kang L, Jian W, Arnoult E, Case M, Jenkinson C, Chi E, Swanson RV, Kievit P, Grove K, Macielag M, Erion MD, SinhaRoy R, and Leonard JN (2019) A Long-Acting PYY Analog Mediates Robust Anorectic Efficacy with Minimal Emesis in Nonhuman Primates. Cell Metabolism 29:837-843.e5.

Richter WF, Grimm HP, Gouy MH, Sogaard S, Kreuzer C, Wessels U, Draganov D, Muenzer C, and Hoche T (2020) Subcutaneous Site-of-Absorption Study with the Monoclonal Antibody Tocilizumab in Minipigs: Administration Behind Ear Translates Best to Humans. The AAPS journal 22:63.

Ryman JT and Meibohm B (2017) Pharmacokinetics of Monoclonal Antibodies. CPT Pharmacometrics Syst Pharmacol 6:576-588.

Schmitt C, Kuhn B, Zhang X, Kivitz AJ, and Grange S (2011) Disease-drug-drug interaction involving tocilizumab and simvastatin in patients with rheumatoid arthritis. Clin Pharmacol Ther 89:735-740.

Schneider DW, Heitner T, Alicke B, Light DR, McLean K, Satozawa N, Parry G, Yoo J, Lewis JS, and Parry R (2009) In vivo biodistribution, PET imaging, and tumor accumulation of $86 \mathrm{Y}$ - and 111 In-antimindin/RG-1, engineered antibody fragments in LNCaP tumor-bearing nude mice. J Nucl Med 50:435-443.

Tabrizi M, Bornstein GG, and Suria H (2010) Biodistribution mechanisms of therapeutic monoclonal antibodies in health and disease. The AAPS journal 12:33-43.

Thomas VA and Balthasar JP (2019) Understanding Inter-Individual Variability in Monoclonal Antibody Disposition. Antibodies 8:56.

Thway TM, Magana I, Bautista A, Jawa V, Gu W, and Ma M (2013) Impact of anti-drug antibodies in preclinical pharmacokinetic assessment. The AAPS journal 15:856-863.

Tibbitts J, Canter D, Graff R, Smith A, and Khawli LA (2016) Key factors influencing ADME properties of therapeutic proteins: $A$ need for $A D M E$ characterization in drug discovery and development. MAbs 8:229-245.

Turner MR and Balu-lyer SV (2018) Challenges and Opportunities for the Subcutaneous Delivery of Therapeutic Proteins. J Pharm Sci 107:1247-1260.

Waldmann TA, Strober W, and Mogielnicki RP (1972) The renal handling of low molecular weight proteins: II. Disorders of serum protein catabolism in patients with tubular proteinuria, the nephrotic syndrome, or uremia. The Journal of Clinical Investigation 51:2162-2174.

Walles M, Berna M, Jian W, Hauri S, Hengel S, King L, Tran J, Wei C, Xu K, and Zhu X (2021) A Cross Company Perspective on the Assessment of Therapeutic Protein Biotransformation. Drug Metab Dispos (in preparation). 
Wang EQ, Plotka A, Salageanu J, Sattler C, and Yunis C (2017) Pharmacokinetics and pharmacodynamics of bococizumab, a monoclonal antibody to PCSK9, after single subcutaneous injection at three sites [NCT 02043301]. Cardiovasc Ther 35: e12278.

Wang Q, Delva L, Weinreb PH, Pepinsky RB, Graham D, Veizaj E, Cheung AE, Chen W, Nestorov I, Rohde E, Caputo R, Kuesters GM, Bohnert T, and Gan LS (2018) Monoclonal antibody exposure in rat and cynomolgus monkey cerebrospinal fluid following systemic administration. Fluids Barriers CNS 15:10.

Wang W, Wang EQ, and Balthasar JP (2008) Monoclonal antibody pharmacokinetics and pharmacodynamics. Clin Pharmacol Ther 84:548-558.

Werle $M$ and Bernkop-Schnurch A (2006) Strategies to improve plasma half life time of peptide and protein drugs. Amino Acids 30:351-367.

Wills RJ (1990) Clinical Pharmacokinetics of Interferons. Clinical Pharmacokinetics 19:390-399.

Xu Z, Wang Q, Zhuang Y, Frederick B, Yan H, Bouman-Thio E, Marini JC, Keen M, Snead D, Davis HM, and Zhou $\mathrm{H}$ (2010) Subcutaneous bioavailability of golimumab at 3 different injection sites in healthy subjects. J Clin Pharmacol 50:276-284.

\section{FOOTNOTES}

Financial Disclosure: No author has an actual or perceived conflict of interest with the contents of this paper. This paper received no external funding. 


\section{FIGURE LEGENDS}

Fig. 1. Examples of Therapeutic Proteins

Fig. 2. Percent response from the participating companies on conducting studies to understand mechanism of absorption, distribution, biotransformation, and elimination routes for therapeutic proteins.

Fig. 3. Results from the survey on most frequently used route of administration (A) and nonclinical species used for PK assessment (B). Although wild-type and transgenic mice are used for PK evaluation, non-human primate is the most frequently used preclinical species for PK assessment.

Fig. 4. Percent response on conducting preclinical and clinical biodistribution studies for TPs.

Fig. 5. Percent responses on utility of biotransformation (BT) data of TPs. Among the companies participated, respondents utilize BT data for explaining PK/PD disconnect, clearance mechanism, and safety aspects.

Fig. 6. Results from the survey on regulatory strategy within the company (A), regulatory interaction experience (B) and need for additional guidance (C). 
TABLE 1. Number of ADME Constituents Presented in BLA Submission Packages (FDA) of Therapeut:iّe Proteins Reviewed from 2011 to 2020

\begin{tabular}{lccccc}
\hline Molecule Types & Total BLAs & Absorption* & Distribution & Metabolism & Excretion \\
\hline Monoclonal Antibodies & 60 & 60 & 8 & 0 & 1 \\
Fusion Proteins & 11 & 11 & 4 & 0 & 0 \\
Enzymes & 8 & 8 & 2 & 0 & 0 \\
Proteins & 12 & 12 & 6 & 1 & 4
\end{tabular}

*Absorption values include mechanisms of absorption and pharmacokinetic evaluations. 
TABLE 2. Techniques and analytical methods used for preclinical and clinical biodistribution studies

\begin{tabular}{llc}
\hline Technique & Preclinical & Human \\
\hline Ligand binding assays, immune staining & yes & yes \\
QWBA using radio label & yes & no \\
Immunocapture LC/MS & yes & yes \\
Imaging (e.g MALDI-MS, PET, fluorescence) & yes & yes
\end{tabular}



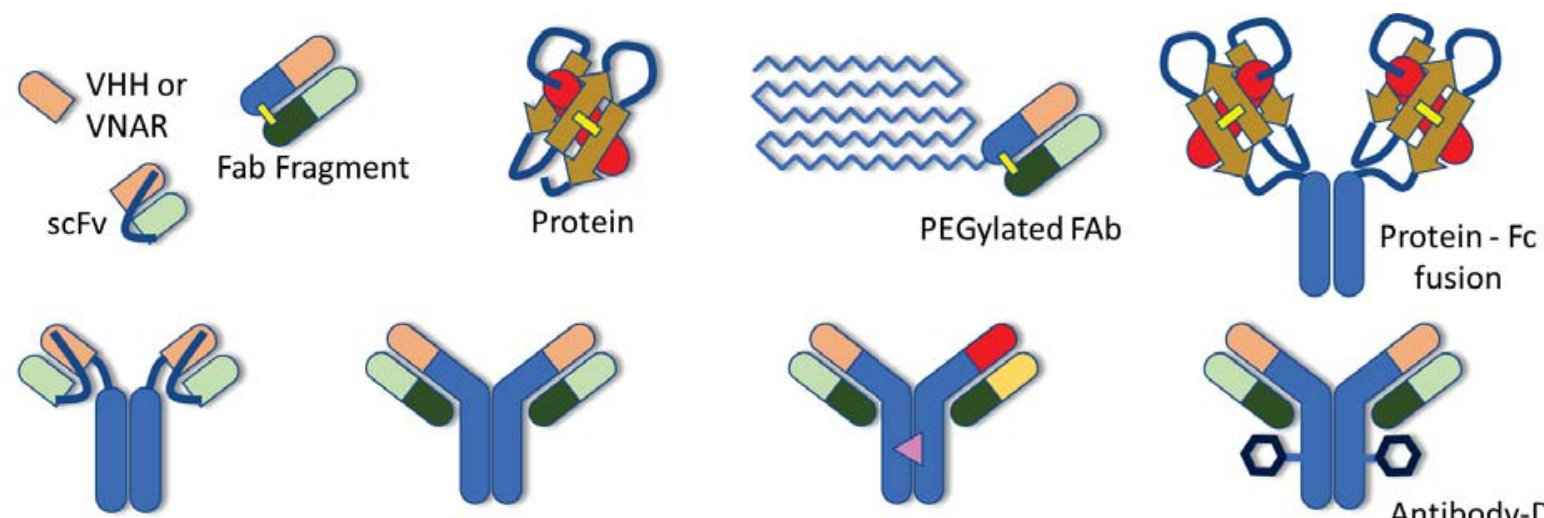

Antibody
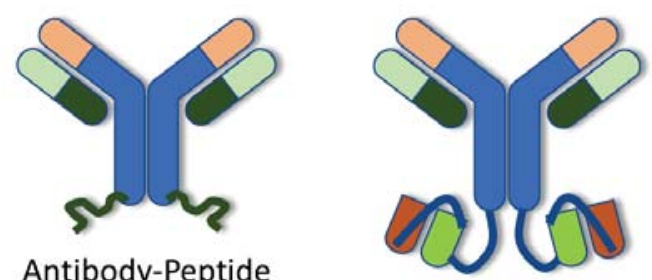

Antibody-scFv fusion
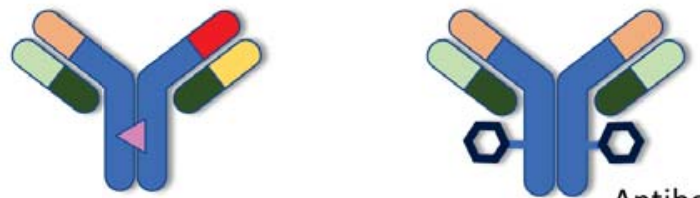

Antibody-Drug

Conjugate
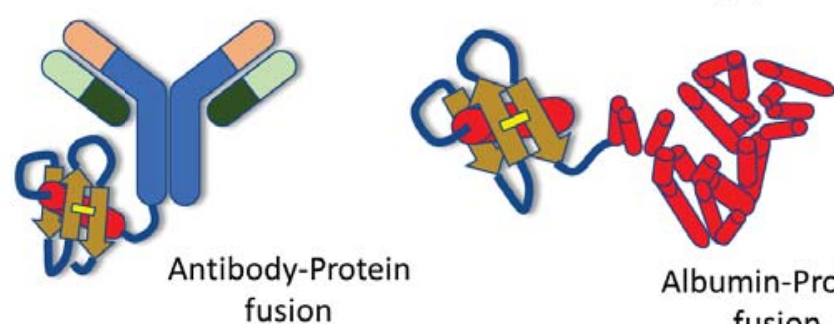

Albumin-Protein fusion

VHH - camelid antibody; VNAR - variable new antigen receptors; PEG - polyethylene glycol; $\mathrm{scFv}$ - single chain variable fragment; Fab - antigen binding fragment; Fc - crystallisable fragment

It should be noted that the diversity of molecules shown in this diagram is not exclusive, and represents known modalities to the authors currently in preclinical or clinical development. Not all these formats have been granted market authorisation.

Figure 1 


\section{Does your company characterize ADME?}

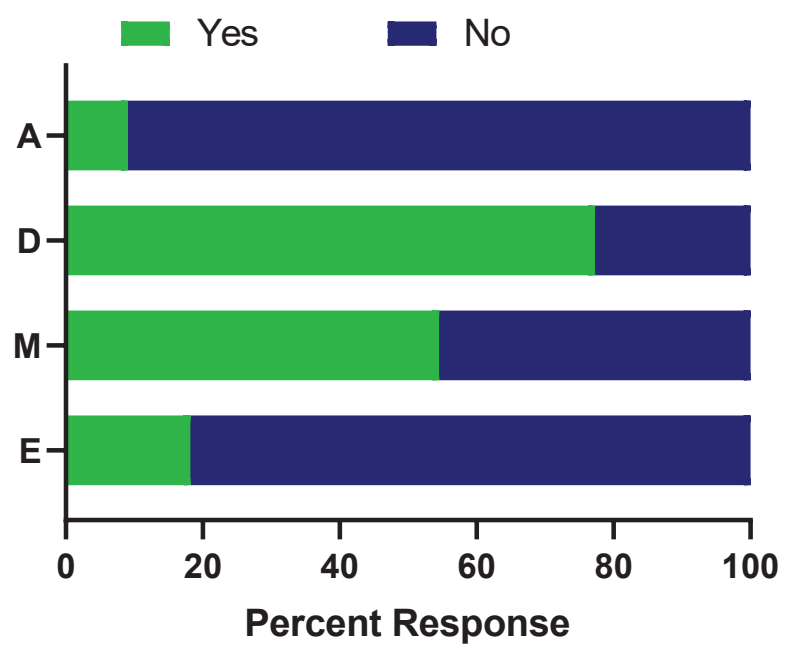

A: Mechanisms of absorption

D: Biodistribution to target tissues in preclinical species

M: Metabolism/Biotransformation

E: Elimination routes 
A Most frequently used route of administration for TPs?

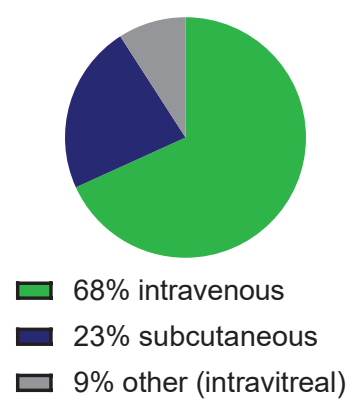

B In vivo models to determine PK of TPs

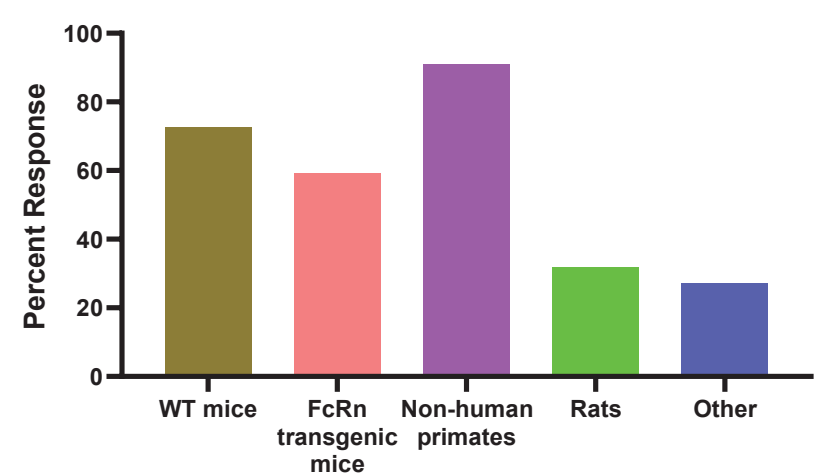

Figure 3 


\section{Does your company perform biodistribution studies?}

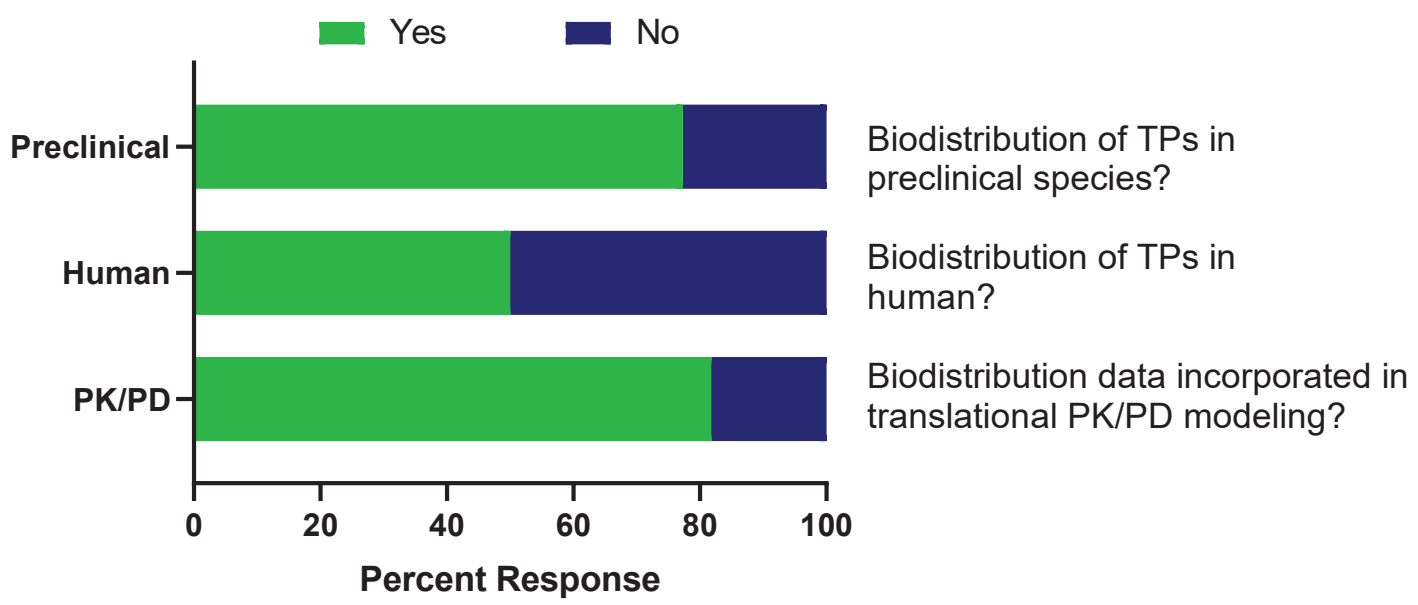

Figure 4 
How is your biotransformation data being utilized?

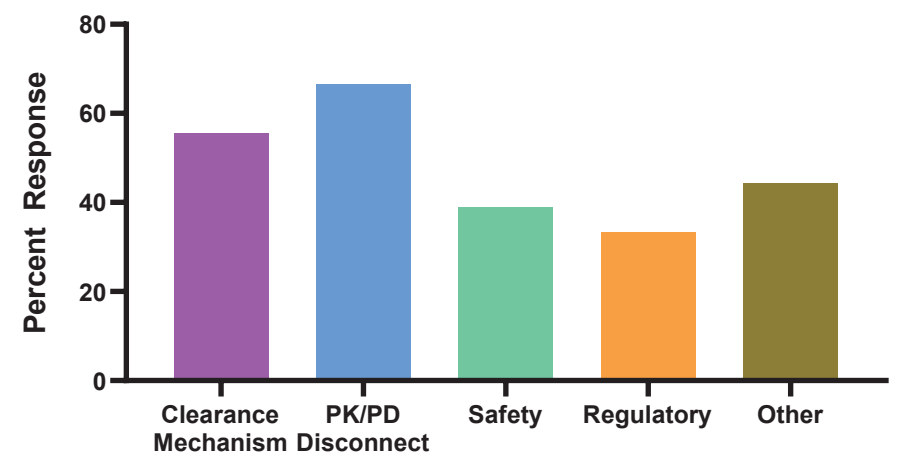

Figure 5 
A Does your company have a regulatory strategy for ADME of TPs?

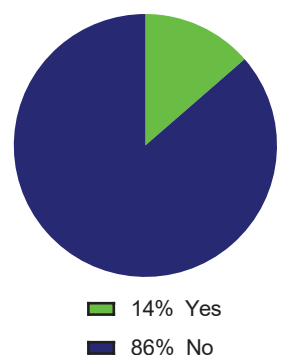

B Have your interaction with regulatory agencies led to conduct additional ADME studies of TPs?

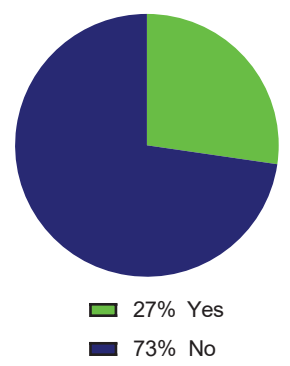

C What is your expectation on the need for further regulatory guidance on ADME of TPs?

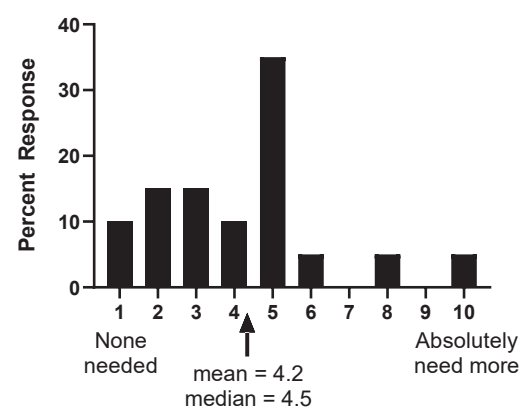

Figure 6 